\title{
A instrução formal da gramática no ensino/aprendizagem de L2: benefícios e propostas
}

\author{
Adriana Maria Tenuta de Azevedo \\ Universidade Federal de Minas Gerais
}

\section{Abstract}

This text aims at dealing with the following questions: "Should we teach grammar?"; "If we should, how can instruction of aspects of the grammatical structure of the target language be of benefit to the learner of L2?". This kind of instruction had been considered 'out of fashion' for some time; however, in the 80's there was a 'rebirth' of the interest in grammar instruction. We will focus on the dichotomy product/process concerning language and grammar, with the intention of arguing in favor of Batstone's pedagogical continuum. Also, we will approach TBLT and C-R, which show a preference for the view of process without neglecting the necessity for precise regulation of the linguistic production of the learner. C$\mathrm{R}$ also considers the notion of the relationship among the grammatical segments of syntax, semantics and discourse as fundamental . 


\section{INTRODUÇÃO}

$\mathrm{E}$

ste trabalho visa a discutir as seguintes questões relacionadas

ao ensino/aprendizagem de língua estrangeira: ${ }^{1}$

- Devemos ensinar gramática?

- Se consideramos que sim, como ensiná-la, ou seja, como pode a instrução de aspectos da estruturação gramatical ser de utilidade para o aprendiz de L2? ${ }^{2}$

Pretende-se discutir a relevância do ensino da gramática, ou seja, da explicitação, para o aprendiz, de aspectos da estrutura gramatical da língua estrangeira. Argumenta-se em favor da instrução de aspectos formais da língua-alvo aliada a um trabalho voltado para a prática em 'uso da língua', para os aspectos comunicativos do aprendizado de língua estrangeira. Apresentam-se propostas de abordagens para essa instrução.

Para isso, primeiro apresentamos de uma visão histórica sintética de como uma situação de supremacia do ensino de gramática através de vários séculos foi seguida por um período no qual esse ensino foi desvalorizado, chegando-se posteriormente ao ressurgimento do interesse pela instrução formal de aspectos gramaticais.

Segundo, abordamos a dicotomia produto/processo no que concerne à visão da língua e da gramática, com o intuito de argumentar-se a favor do contínuo pedagógico, que propõe que o maior ou menor controle da produção lingüística do aprendiz depende da situação e do momento da aprendizagem.

Terceiro, focalizamos a proposta TBLT (Task Based Language Teaching) e a abordagem C-R (Consciousness-Raising), que adotam preferencialmente a visão de processo, sem negligenciar a necessi- 
dade de uma certa regulamentação da produção lingüística do aprendiz. Para C-R é ainda fundamental a noção da interrelação entre os segmentos gramaticais da sintaxe, semântica e discurso.

\section{SUPREMACIA, DECLÍNIO E RETORNO AO ENSINO DE GRAMÁTICA}

TONKYN (1994) faz um levantamento histórico, mostrando como os ramos emergentes dos estudos lingüísticos, tais como a sociolingüística, a psicolingüística, o movimento communicative language teaching, por exemplo, contribuíram para haver um período no qual o ensino da gramática perde a posição de destaque que ocupara através dos séculos com a tradição do ensino de língua dominada pelo latim e pelo grego. O autor analisa a evolução dos estudos lingüísticos a partir do Método Gramática-Tradução. Segundo ele, na primeira metade do século vinte, os sucessores desse método passam a refutar o foco que se dava ao conhecimento gramatical e à tradução como meio de ensino.

Apesar disso, a lingüistica estrutural, segundo Tonkyn, mantinha ainda, como dever seu, a transmissão do sistema gramatical.

"It is the aim of the linguist to reveal the system of the language, the langue, and of the language teacher to enable people to learn it." (WILKINS, 1972: 68 apud TONKYN, 1994:2)

Já a revolução chomskiana questiona a adequação da abordagem estruturalista, porém mantém também a gramática no centro do interesse lingüístico. As idéias de Chomsky não tiveram um efeito direto sobre o ensino de língua, mas possibilitaram o renascimento das abordagens pedagógicas mentalistas e cognitivas.

No final dos anos 50 e início dos anos 60, os professores de língua encontravam-se em um dilema, uma vez que a lingüística ainda não oferecia uma abordagem científica da gramática que lhes fosse útil. Primeiro, por não se ter chegado a um consenso quanto ao melhor método de análise gramatical. Segundo, porque as descrições lingüísticas eram inacessíveis aos professores. Esses 
tornam-se céticos quanto à relevância dos estudos gramaticais para a sua prática. A evolução da lingüística até aquele momento gerou, então, um efeito equivocado quanto ao status da gramática no ensino de língua.

"On the one hand, traditional grammar was supposed to be
'unscientific' and therefore unworthy of serious consideration, while
linguistics seemed to be a highly esoteric subject beyond the
comprehension of any but the most dedicated of University
scholars... As a result many teachers became disillusioned, not only
about modern linguistics, but about linguistics in general, including
traditional grammar, and there was a widespread reaction against
grammar-teaching in schools." (Allen and Widdowson, 1975: 45
apud TONKYN 1994:3)

A sociolingüistica foi também, segundo Tonkyn, de grande influência para o "destronamento" da gramática no ensino de língua. O descritivismo, em oposição ao prescritivismo, bem como a ênfase na língua falada, já afirmados pela lingüística estrutural, com a sociolingüística levam a uma alteração da noção de "correção" na qual se baseavam as antigas gramáticas escolares. O sociolingüista Hymes, nesta época, propõe a ampliação da noção de "competência" chomskiana, no sentido de uma "competência comunicativa". O conceito reformulado expressa agora a habilidade de se usar e interpretar a língua apropriadamente em contextos específicos (HYMES, 1972 apud TOMKYN, 1994).

Outro fator de desvalorização do ensino da gramática foi o movimento Communicative Language Teaching, que, visando a reproduzir em sala de aula traços de uso real da língua, não encarava a precisão gramatical como imprescindível ao sucesso comunicativo.

A psicolingüística também contribuiu para o declínio da importância da gramática na metodologia e estruturação do curso de língua estrangeira. A concepção de Chomsky do mecanismo inato de aquisição de linguagem, aliada a estudos que revelavam regularidades na ordem de aquisição de traços morfológicos, constituiu uma base importante para Hipótese da Evidência (Input Hypothesis) de Krashen (KRASHEN, 1985 apud TONKYN, 1994). A 
visão de Krashen do processo de aquisição de segunda língua, segundo o qual o mecanismo inato operaria sob as condições corretas de input compreensível, marginalizou o papel da instrução baseada na forma.

Essa era a situação no início dos anos 80 tanto para o ensino de L1, como para o de L2, especialmente na Inglaterra e nos Estados Unidos: o ensino da gramática havia perdido a posição central que desfrutara anteriormente. Entretanto, ele nunca foi de fato totalmente abandonado. Muitos professores, por "conservadorismo ou ecletismo sábio", como afirma Tonkyn, mantiveram a tradição, mesmo quando esta estava oficialmente "fora de moda".

É na segunda metade dos anos 80 que acontecerá na Inglaterra um "retorno à gramática" tanto no ensino L1, como no de L2. Nessa nova fase, ao invés de argumentar-se a favor ou contra o ensino da gramática, os pesquisadores começaram a investigar as situações para as quais essa instrução traria benefícios.

E esses benefícios são vários, como veremos a seguir ao abordarmos a dicotomia produto/processo como visões de língua e de gramática.

\section{PRODUCT AND PROCESS: LÍNGUA E GRAMÁTICA}

BATSTONE (1994) lembra-nos que uma das características da gramática é o fato de se poder considerá-la de diferentes pontos de vista.

Há, por um lado, visões da língua como produto, ou seja, como sendo estruturada e sistemática, composta de partes.

Por outro lado, ao invés de pensarmos em termos de um conjunto de formas separadas, podemos pensar a gramática como dinâmica; uma fonte explorada pelos usuários da língua ao construírem seu discurso. Esse ponto de vista tem a ver com movimento, mudança, e com os inúmeros fatores que condicionam o contínuo rearranjo da gramática "em ação". Esta é uma perspectiva da língua como processo. 
No que diz respeito ao ensino, a distinção ocorre entre um controle cuidadoso das formas lingüísticas para o aprendiz (como produto), e o uso criativo da língua pelo aprendiz (como processo).

Há, pelo que depreendemos das colocações de BATSTONE (1994), aspectos positivos e negativos para o ensino em se adotando exclusivamente qualquer uma das visões.

\section{Produto: aspectos negativos}

1. Segundo BATSTONE (1994), pesquisa em aquisição de segunda língua demonstra que o aprendiz não passa diretamente de um conhecimento zero à proficiência total de uma determinada estrutura gramatical. O que acontece no processo de aprendizagem é que hipóteses sobre o funcionamento da estrutura vão sendo constantemente reformuladas em direção à forma ideal (She go office pode transformar-se em she goed office antes da manifestação da forma apropriada). (KELLERMAN, 1985; MCLAUGHLIN, 1990 apud BATSTONE, 1994).

Quando ensinamos gramática como produto, dizem que estamos confrontando o aprendiz com formas-alvo que não poderão, num primeiro encontro, ser "estruturadas" dentro das suas hipóteses correntes.

2. Declara-se freqüentemente que muitos syllabuses de produto trabalham com a memorização, o que leva a um modo superficial de aprendizagem, no qual os aprendizes apenas "acumularão" os itens de língua (WIDDOWSON, 1979: 284; RUTHERFORD, 1987: 154-5 apud BATSTONE, 1994).

3. O ensino de gramática como produto desconsidera o fator "uso da língua". Instruído apenas através de uma abordagem voltada para a forma, o aprendiz pode não reter aquilo que tenha acabado de aprender. Algumas formas cuidadosamente praticadas podem ser rapidamente esquecidas. 


\section{Produto: aspectos positivos}

1. Argumenta-se que, antes de começar a estruturar a língua em "hipóteses", os aprendizes precisarão notar, de forma explícita, determinados aspectos da gramática (SCHMIDT, 1990 apud BATSTONE, 1994), de forma que "notar a gramática" pode ser um estágio preliminar no processo de aprendizagem. Quanto ao processo de estruturação e reestruturação de hipóteses, o ensino da língua como produto pode ser benéfico, no sentido de que os aprendizes terão várias chances de comparar suas hipóteses correntes com os modelos alvos disponíveis no input.

2. Outra maneira de justificar o ensino de gramática como produto é dizer que fornece ao aprendiz um quadro claro e explícito (pois as estruturas são apresentadas step by step). Tal abordagem estruturada e sistemática pode gerar às vezes um sentimento de segurança e propósito, que é motivador.

\section{Processo: aspecto positivo}

A fim de garantir-se uma efetiva internalização da gramática, é necessário ter-se uma prática considerável no "uso da língua" (language use), focalizando o significado e a auto-expressão, capacitando o aprendiz a lidar com o ritmo das interações que acontecem em tempo real.

\section{Processo: aspecto negativo}

No trabalho com gramática como processo, o foco está no significado e na auto-expressão do aprendiz. Entretanto, a ênfase em atividades de processo mal-concebidas, nas quais as respostas não são apropriadamente antecipadas, pode levar a uma "abdicação", por parte do professor, de qualquer tipo de controle da produção lingüística do aprendiz. Essa situação tende a resultar na internalização de língua gramaticalmente empobrecida. 


\section{O "CONTíNUO PEDAGÓGICO"}

Como visto acima, qualquer um dos dois modelos tem aspectos positivos e negativos. O que parece, então, desejável, é um grau de equilíbrio entre o que são esses dois extremos. BATSTONE (1994) afirma que esse equilíbrio pode ser conseguido através de uma concepção de ensino de gramática que ofereça uma certa regulamentação da atividade do aprendiz, mas que não desconsidere a necessidade de prepará-lo para os processos do uso da língua. O autor diz que devemos pensar em processo e produto não como polaridades, porém, como pontos em um "contínuo pedagógico", como representado abaixo:

\section{A pedagogic continuum}

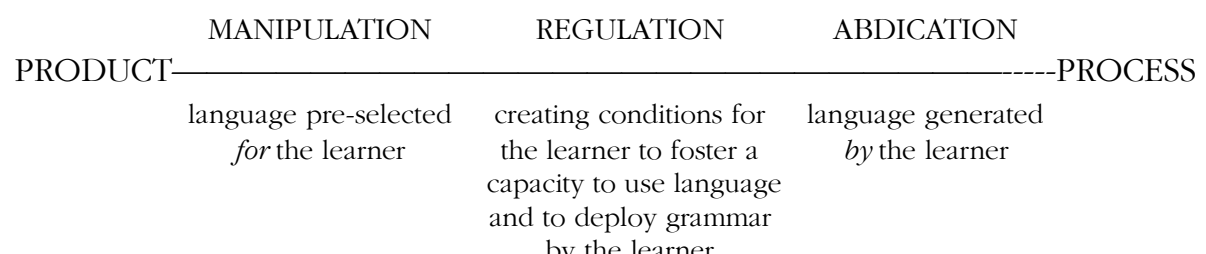

(BATSTONE, 1994: 229)

O autor afirma que tem havido, nos últimos dez anos, propostas que se baseiam nessa noção de que a produção do aprendiz requer alguma forma de controle (regulation). Uma delas é a TaskBased Language Teaching (TBLT), como concebida por Crooks e Long (1987a, 1987b, 1992 apud BATSTONE, 1994). Em TBLT há muita ênfase no "uso da língua" através da interação. Com o tipo correto de controle e design, tais tasks podem encorajar os aprendizes a ampliar ( $s t r e c h$ ) sua capacidade de utilização da língua-alvo (LONG 1985, 1989 apud BATSTONE, 1994), superando os limites de suas habilidades correntes. 
Abaixo, estão alguns modelos interacionais que têm essa finalidade:

“Two-way tasks: two-way exchange of information between interlocutors.

'Closed' tasks: where learners know they have to reach a predetermined correct solution (should lead to greater quantity and quality of negotiation than 'open' tasks).

Diverging'tasks: in which learners take up divergent views in a particular issue, as with a debate- may lead to production which is quite complex and elaborate."

(BATSTONE, 1994: 230)

Naturalmente não é dito que tais tasks levam automaticamente a uma produção lingüística mais rica. É preciso também proporcionar aos aprendizes prática nas várias habilidades envolvidas no uso da língua e guiá-los na habilidade de usar a gramática para fins comunicativos.

Uma medida nesse sentido de habilitar o aprendiz a utilizar a gramática comunicativamente é a abordagem de ensino que enfoca o processo de grammaticisation.

\section{O ENSINO DE GRAMÁTICA COMO GRAMMATICISATION}

Esta abordagem, que teve suas origens nos trabalhos de RUTHERFORD (1987) e WIDDOWSON (1990), considera que os aprendizes partem de palavras, que eles combinam e modificam a partir da aplicação da gramática. Há teorias sobre aquisição de L1 e L2 que indicam que, primeiramente, a linguagem do aprendiz é tipicamente lexical, isto é, as palavras são sobrepostas umas às outras com um mínimo de coesão gramatical, a fim de emitir uma significação básica. (PETERS, 1983; Nattinger e de Carrico, 1992 apud BATSTONE 1994). Segundo essa visão, a gramática é aprendida gradualmente, partindo da base de palavras, através de um processo de reestruturação.

Alguns pesquisadores defendem que todo o processo de desenvolvimento da interlíngua pode ser visto como um movimento 
gradual do léxico para a gramática, envolvendo uma mudança progressiva em direção a um "modelo mais sintático" (GIVÓN, 1979 apud BATSTONE, 1994), sendo que uma das principais motivações para esta evolução é a motivação comunicativa. Com relação a isso, temos:

"The greater the contribution of context in the sense of shared knowledge, the less need there is for grammar to augment the association of words... The less effective words are in identifying relevant features of context... the more dependent they become on grammatical modification of one sort or another ... grammar is not a constraining imposition but a liberating force: It frees us from a dependency on context." (WIDDOWSON, 1990: 86 apud BATSTONE, 1994: 231)

Tasks propostos para grammaticisation têm o objetivo não de apresentar a gramática como produto pré-formulado, com as escolhas feitas por outra pessoa, mas sim de reproduzir em contextos de ensino a maneira como a utilização da gramática acontece em situações reais de comunicação. A gramática a ser utilizada pelo aprendiz surgirá como resultado de suas próprias escolhas.

A concepção de gramática que Rutherford defende em sua proposta de Consciousness-Raising (C-R), relacionada à abordagem grammaticisation, está expressa na citação que se segue:

"Grammar should be seen as the 'on-line processing component of discourse, and not the set of syntactic building blocks with which discourse is, as it were, constructed." (RUTHERFORD, 1987: 104 apud BATSTONE, 1994: 233)

Rutherford, portanto, acredita na necessidade de haver um tipo formal de instrução e propõe C-R, que irá ajudar o aprendiz a construir um modelo de referência explícito e será útil no sentido de:

- iluminar aspectos problemáticos do sistema de L2;

- prover evidência contrastiva com a L1 do aprendiz;

- prover evidência negativa de como o sistema não funciona;

- restringir as hipóteses do aprendiz a respeito do sistema de L2. 
Em relação à evidência negativa mencionada acima, JAMES (1994) apresenta a discussão teórica que parte da afirmação de Chomsky de que as crianças aprenderiam a língua apenas através da evidência positiva (indireta), ou seja, da exposição a discurso semanticamente rico; possivelmente também aprenderiam, notando o que não é dito (evidência negativa indireta). Nos círculos de ensino de língua estrangeira, a utilidade de ambas as formas de evidência negativa é mais amplamente aceita.

Em situação de ensino-aprendizagem o fator que gera evidência negativa direta é a resposta a erro (explicando por que certa forma está errada em vez de simplesmente dizer que está errada). RUTHERFORD (1987: 161) diz que é muito melhor que o que tiver de ser julgado emane do próprio aprendiz, de sua própria produção. Da mesma forma, PRAHBU (1987: 76 apud MITCHELL, 1994) insiste que a atenção em sala de aula deve ser dada às formas lingüísticas "auto-iniciadas, não-planejadas, previstas ou controladas pelo professor". Isso significa que a explicação gramatical deve ter o objetivo de remediar, ficando implícito que os textos pedagogicamente autênticos são aqueles produzidos pelos próprios aprendizes e não por falantes nativos.

Em relação à evidência contrastiva, mencionada também como aspecto positivo de C-R, considera-se que o conhecimento da L2 pode ser relacionado e integrado ao conhecimento consciente da L1, como um primeiro passo em direção à automatização do conhecimento da L2. Essa visão procura construir ligações entre o "conhecimento" da L1 e a "ignorância" da L2, sem preocupação excessiva com a interferência da NL.

RUTHERFORD(1987) analisa aspectos característicos da interlíngua de aprendizes da língua inglesa como L2 e enfatiza a relevância de se trabalhar com estes aprendizes para levá-los à conscientização das diferenças existentes entre essa interlíngua e a língua alvo.

Para Rutherford, a visão da língua como processo pressupõe, então, a visão da interrelação entre o discurso, a semântica e a sintaxe. Esta concepção de Consciousness-Raising é consistente com o princípio top-down de funcionamento gramatical. 
A sentença vista como mensagem apresenta uma estrutura de informação e também uma estrutura temática. A estrutura de informação é a distribuição dos elementos dados e novos. Já a estrutura temática apresenta a sentença como composta de um tema, que é o elemento que inicia a proposição, e o rema, que contém o restante dos elementos da sentença.

A escolha temática pode ser variada, mas é determinada contextualmente. Para atendermos a um determinado princípio discursivo (estrutura de informação dado/novo, por exemplo) fazemos uma escolha lexical para tema, que vai gerar processos sintáticos de movimentos de constituintes na frase, a utilização de uma estrutura passiva, ou ainda uma estrutura cleft ou pseudo-cleft. Observe:

a) Martha brought the parcel yesterday.

b) The parcel was brought by Martha yesterday.

c) It was Martha who brought the Parcel yesterday.

d) Yesterday Martha brought the parcel.

e) Yesterday it was Martha who brought the parcel.

Podemos notar que, apesar de serem semanticamente muito semelhantes, as proposições acima não se encaixam todas exatamente nos mesmos contextos. Por exemplo, em resposta a What did Martha bring yesterday? as proposições (a) e (d) são as mais indicadas. Em outro contexto, What about the parcel? a escolha temática apropriada seria parcel, por exemplo, gerando a estrutura passiva (b).

Por exemplo, a interlíngua do aprendiz é geralmente caracterizada por apresentar os constituintes da sentença em sua posição básica, não-marcada. O aprendiz, em fase inicial de aprendizado, em geral, não aplica regras de movimento de constituintes, utilizará uma mesma estrutura, mesmo que o contexto exiga variação; há, na sua produção lingüística um "alinhamento" entre semântica e sintaxe, isto é, as relações semânticas dos enunciados são expressas diretamente pelas posições sintáticas. 
O trabalho que é proposto dentro da abordagem de Consciousness-Raising (C-R), que trabalha com a grammaticisation, é a conscientização desses fatos. As atividades de C-R não constituem a totalidade do trabalho do ensino/aprendizagem de L2. Devem servir, entretanto, para sensibilizar os aprendizes para a influência primordial do discurso na ordenação linear dos blocos de informação.

C-R não é um fim em si mesmo. É um meio para se atingir um fim. O que se torna consciente não é um produto, mas aspectos do processo gramatical e, nessa concepção, ensinar uma língua não é ensinar um corpo de conhecimento, mas sim ensinar como aprender. Há aqui certamente uma diferença em relação às abordagens que levam à memorização de estruturas ou de mecanismos de transformação. Ao conscientizar-se de determinados princípios e processos, o aprendiz torna-se sensível aos contextos lingüísticos e habilitado a uma produção mais apropriada, contextualmente falando.

\section{CONCLUSÃO}

Vimos que o ensino da gramática no processo de aprendizagem de língua estrangeira perdeu a posição de centralidade que ocupara por vários séculos, nos métodos que se baseavam na tradição, no ensino de grego e latim e passou a dividir com o ensino de outras habilidades a atenção que recebia com exclusividade. Ensinar gramática chegou mesmo a estar oficialmente "fora de moda", como vimos em TONKYN (1994), apesar de nunca terem os professores deixado totalmente de fazê-lo.

Essa desvalorização ou o questionamento em relação ao ensino da gramática ocorreu especialmente por causa da evolução dos estudos lingüísticos. Como discute Tonkyn, se por um lado essa evolução contribuiu para a elucidação de aspectos diferenciados da linguagem e sua utilização, por outro teve um efeito, em alguns casos, indesejáveis, sobre o professor de língua, que, muitas vezes, por obter apenas informações parciais de estudos emergentes, 
confundiu-se ao ver sua prática ser negada ou desvalorizada e não poder de imediato compreender o que poderia substituí-la.

Mais recentemente, surge uma situação interessante com a possibilidade de conciliar-se a tradição ao novo. Tradição é a visão de língua e gramática como produto, tendo a gramática, no processo de ensino/aprendizagem, posição de destaque. O novo é a concepção de que se aprende a usar uma língua usando-a, ou seja, de que é extremamente necessária a prática de aspectos de "uso da língua", com ênfase no significado, na auto-expressão, e não mais apenas na forma. O aprendiz é visto agora como um indivíduo que necessita comunicar-se.

Há benefícios no ensino da gramática, como vimos através das colocações de Tonkyn, Batstone, Rutherford, que são, por exemplo: proporcionar a evidência negativa, mostrando ao aprendiz como o sistema não funciona; salientar aspectos da estruturação da línguaalvo, dando a chance de ele checar constantementes suas hipóteses; prover contexto para a prática de uma forma específica; ajudar a evitar a fossilização prematura que uma ênfase excessiva na performance de tarefas comunicativas pode causar.

Entretanto, devemos considerar também a necessidade das atividades que tentem reproduzir situações de comunicação reais, com foco na mensagem (message focus, ou form de-focus, como argumenta JOHNSON, 1994).

Tanto a prática de aspectos gramaticais, quanto a vivência de situações de comunicação o mais reais possíveis são necessárias aos aprendizes de língua estrangeira, uma vez que não há situação de imersão e eles não dispõem de input suficiente para um rápido aprendizado. A instrução de aspectos gramaticais é necessária, especialmente para aprendizes adultos, até porque já passaram por um processo de instrução formal em língua materna e podem beneficiar-se de um contato metacognitivo com aspectos de L2.

Certamente o "como ensinar" é muito importante. A visão de Batstone do "contínuo pedagógico", no qual se encaixam TBLT, gramaticisation e Consciouness-Raising, com a perspectiva de uma 
regulamentação variável da produção do aprendiz, acena com a possibilidade da resolução daquele conflito em que se viram muitos professores. O contínuo produto/ processo representa uma opção para aqueles que não desejam manter-se inflexíveis em sua didática, utilizando uma única abordagem de forma exclusiva. Argumenta-se, então, em favor de uma metodologia mista, com mecanismos de ensino variados, na qual coexistam o trabalho com gramática como processo e atividades mais voltadas à visão de produto. Cabe ao professor e ao aluno, que também deve assumir responsabilidade nesse processo de ensino/aprendizagem, aferirem a efetividade de qualquer abordagem utilizada.

\section{NOTAS}

${ }^{1}$ Este trabalho pretende discutir a relevância do ensino da gramática, ou seja, da instrução, ou explicitação de aspectos da estrutura gramatical da língua estrangeira exclusivamente. Não pretende discutir questões relacionadas ao ensino/ aprendizagem de língua materna.

${ }^{2} \mathrm{~L} 2$, neste trabalho, refere-se tanto à língua estrangeira, quanto à segunda língua.

\section{REFERÊNCIAS BIBLIOGRÁFICAS}

BATSTONE, R. Grammar in the second language classroom. In: Grammar and the second language teacher. UK: Prentice Hall, 1994.

CANALE, M. From communicative competence to communicative language pedagogy. In: Language and Communication, Longman, 1983.

DOWNING, A. and LOCKE, P. A university course in English Grammar, Prentice Hall International, 1992.

HALLIDAY, M. A. K. An Introduction to Functional Grammar, London: Arnold, 1985.

JAMES, C. Explaining grammar to its learners. In: Grammar and the second language teacher. UK: Prentice Hall, 1994.

JOHNSON, K. Teaching declarative and procedural knowledge. In: Grammar and the second language teacher. UK: Prentice Hall, 1994. 
MacCARTHY, M. Discourse analysis for language teachers. Cambridge: Cambridge University Press, 1991.

MITCHELL, R. Foreign language teachers and the teaching of grammar. In: Grammar and the second language teacher. UK: Prentice Hall, 1994.

RICHARDS, J. C. The language taching matrix, Cambrige University Press, 1990.

RUTHERFORD, W. Second language grammar: learning and teaching, Longman, 1987.

TERENCE, O. Chapter 1: Introduction. In: Perspectives on Pedagogical Grammar, Cambridge University Press, 1994.

TONKYN, A. Introduction: Grammar and the second language teacher. In: Grammar and the second language teacher. UK: Prentice Hall, 1994. 